\title{
Article \\ Discontinuous Galerkin Isogeometric Analysis of Convection Problem on Surface
}

\author{
Liang Wang ${ }^{1}$, Chunguang Xiong ${ }^{1, * \mathbb{D}}$, Xinpeng Yuan ${ }^{2}$ and Huibin $\mathrm{Wu}^{1}$ \\ 1 Department of Mathematics, Beijing Institute of Technology, Beijing 100081, China; \\ lueguang@bit.edu.cn (L.W.); huibinwu@bit.edu.cn (H.W.) \\ 2 State Key Laboratory of Severe Weather, Chinese Academy of Meteorological Sciences, China Meteorological \\ Administration, Beijing 100081, China; yuanxp@cma.gov.cn \\ * Correspondence: xiongchg@bit.edu.cn
}

check for updates

Citation: Wang, L.; Xiong, C.; Yuan, X.; Wu, H. Discontinuous Galerkin Isogeometric Analysis of Convection Problem on Surface. Mathematics 2021, 9, 497. https://doi.org/10.3390/ math9050497

Academic Editor: Carlo Cattani

Received: 20 January 2021

Accepted: 25 February 2021

Published: 28 February 2021

Publisher's Note: MDPI stays neutral with regard to jurisdictional claims in published maps and institutional affiliations.

Copyright: (C) 2021 by the authors. Licensee MDPI, Basel, Switzerland. This article is an open access article distributed under the terms and conditions of the Creative Commons Attribution (CC BY) license (https:// creativecommons.org/licenses/by/ $4.0 /)$.

\begin{abstract}
The objective of this work is to study finite element methods for approximating the solution of convection equations on surfaces embedded in $\mathbb{R}^{3}$. We propose the discontinuous Galerkin (DG) isogeometric analysis (IgA) formulation to solve convection problems on implicitly defined surfaces. Three numerical experiments shows that the numerical scheme converges with the optimal convergence order.
\end{abstract}

Keywords: convection problem; IgA-DG; SPDEs

\section{Introduction}

Surface partial differential equations (SPDEs) arise in natural sciences and applied areas, such as minimal surface equation, Willmore flow, transport of surfactants along interfaces in multiphase fluids [1], and lipid interactions in cell membranes [2]. In this paper, we consider the DG-IgA methods for the following model problem:

$$
\nabla_{\Gamma} \cdot(\beta u)+\gamma u=f,
$$

where $\nabla_{\Gamma^{*}}=\operatorname{div}_{\Gamma}$ denotes the surface divergence, $\Gamma$ is a smooth two-dimensional surface without boundary embedded in $\mathbb{R}^{3} . \beta=\left(b_{1}, b_{2}, b_{3}\right)$ is the advective velocity with $b_{i} \in$ $W_{\infty}^{1}(\Gamma), \gamma \in L^{\infty}(\Gamma)$ is reaction coefficient. We call this model problem the convection equation on surface $\Gamma$. The problem (1) has a unique solution $u \in H(d i v, \Gamma)$ satisfying $\int_{\Gamma} u d \sigma=0$, where $d \sigma$ is surface measure. Moreover, for source term $f \in L^{2}(\Gamma)$ we have $\int_{\Gamma} f d \sigma=0$.

To ensure the existence and uniqueness of a solution $u \in H^{1}(\Gamma)$ to the model problem (1.1), we adopt the following hypothesis: There exists a positive constant $\gamma_{0}$ such that

$$
\gamma(x)+\frac{1}{2} \nabla_{\Gamma} \cdot \beta(x)=\left(c_{0}(x)\right)^{2} \geq \gamma_{0}, \quad \text { for all } x \in \Gamma .
$$

Finite element methods of SPDEs have been extensively developed. G. Dziuk [3] first used the finite element method for solving the surface elliptic problem. A. Bonito and J. E. Pasciak [4] designed and analyzed multigrid algorithms for the Laplace-Beltrami operator on a smooth and closed surface. DG methods for solving the first-order hyperbolic problems were defined in [5-8]. Since DG methods have well-known stability and local conservation in numerical application of partial differential equation problems $[9,10]$ and capture solution blow-ups, it is natural to extend the DG approximation to PDEs on surfaces or manifolds. Ref. [11] extended the discontinuous Galerkin (DG) methods to the first-order hyperbolic and advection-dominated problems on surfaces. 
IgA has been introduced in [12] as a new tool for solving numerically PDEs with the complicated geometric domains, in particular, surfaces. DG-IgA methods have been applied for approximating solutions of elliptic problems on surfaces [13,14]. Ref. [15] developed DG-IgA numerical schemes for solving problems on segmentations with gaps. Ref. [16] studied NURBS-based isogeometric analysis for the computation of flows about rotating components. In [17], a new stabilized symmetric Nitsche method was proposed for enforcement of Dirichlet boundary conditions for elliptic problems in Cut-IgA. The remainder of the paper is organized as follows: First, we introduce some preliminaries about IgA and discrete NURBS finite space $V(h)$. Then we derive the DG-IgA scheme of convection problem (1.1). Finally, we present three numerical experiments to illustrate the discrete formulation.

\section{Model Problem and Discretization}

\subsection{Differential Operators on Surfaces}

Let us consider a two-dimensional surface $\Gamma \subset \mathbb{R}^{3}$ defined in the physical space $\mathbb{R}^{3}$. Assume that the surface is characterized by a geometrical mapping from a parameter space $\widehat{\Gamma} \subset \mathbb{R}^{2}$. Let $X \in C^{2}\left(\widehat{\Gamma}, \mathbb{R}^{3}\right)$ be a local parameterization of $\theta \in \widehat{\Gamma}$, where the vector-valued independent variable $\theta=\left(\theta_{1}, \theta_{2}\right)$ is called parametric coordinate. Indeed, $X$ defines the geometrical mapping as follows:

$$
X: \widehat{\Gamma} \rightarrow \Gamma \subset \mathbb{R}^{3}, \quad \theta \rightarrow X(\theta) .
$$

To define surface gradient, we introduce the Jacobi matrix $J(\theta)=\left(J_{i j}\right)_{3 \times 2}$ as

$$
J(\theta)=\left[\frac{\partial X}{\partial \theta_{1}}, \frac{\partial X}{\partial \theta_{2}}\right] .
$$

So, the metric tensor $G(\theta)=\left(g_{i j}\right)_{2 \times 2}$ is represented by

$$
g_{i j}(\theta)=J(\theta)^{T} J(\theta)=\frac{\partial X(\theta)}{\partial \theta_{i}} \frac{\partial X(\theta)}{\partial \theta_{j}}, i, j=1,2 .
$$

The inverse of $G(\theta)$ is denoted by $G^{-1}(\theta)=\left(g^{i j}\right)_{2 \times 2}$. The tangential or surface gradient is given by

$$
\nabla_{\Gamma} u(X(\theta))=G^{-1}(\theta) \frac{d u(X(\theta))}{d \theta} .
$$

So, we can deduce the tangential or surface divergence. For $v$ is a $C^{1}$ vector field on $\Gamma$, we have

$$
\nabla_{\Gamma} \cdot v=\frac{1}{\sqrt{|G|}}\left(\frac{\partial}{\partial \theta_{1}}, \frac{\partial}{\partial \theta_{2}}\right)\left(\sqrt{|G|} G^{-1} J^{T} v\right)
$$

Remark that the vector field $v$ may not be tangential to surface $\Gamma$. However, the 2-dimensional vector $G^{-1} J^{T} v$ is the projection of $v$ in parametric coordinate $\theta$, which is a tangent vector field of $\Gamma$. So, the definition of surface divergence is well-posed.

Let $L^{2}(\Gamma)$ denote the usual $L^{2}$-space on the surface $\Gamma$ with norm

$$
\|f\|_{L^{2}(\Gamma)}=\left(\int_{\Gamma} f^{2} d \sigma\right)^{\frac{1}{2}} .
$$

Furthermore, we use standard notations $W_{p}^{m}(\Gamma)$ and $H^{m}(\Gamma)$ for Sobolev spaces on $\Gamma$ with norm and semi-norm 


$$
\begin{aligned}
\|f\|_{W_{p}^{m}(\Gamma)}^{p} & =\sum_{k=0}^{m}\|f\|_{W_{p}^{k}(\Gamma)}^{p} \text { and } \quad\|f\|_{W_{p}^{m}(\Gamma)}=\left\|D_{\Gamma}^{m} f\right\|_{L^{p}(\Gamma)}, \\
\|f\|_{H^{m}(\Gamma)}^{2} & =\sum_{k=0}^{m}\|f\|_{H^{k}(\Gamma)}^{2} \text { and } \quad\|f\|_{H^{k}(\Gamma)}=\left\|D_{\Gamma}^{k} f\right\|_{L^{2}(\Gamma)} .
\end{aligned}
$$

To present the weak form of problem (1), we introduce the Stokes theorem on surface $\Gamma$. Let $v$ be a $C^{1}$ vector field on surface $\Gamma, q \in C^{1}(\Gamma)$, then the following identity holds:

$$
\int_{\Gamma}\left(v \cdot \nabla_{\Gamma} q+q \nabla_{\Gamma} \cdot v\right) d \sigma=\int_{\partial \Gamma} q v \cdot n d s
$$

\subsection{Isogeometric Analysis}

To apply the IgA methodology of the problem (1), the domain $\Gamma$ is partitioned into some closed sub-patches such that

$$
\Gamma=\bigcup_{i=1}^{N} \Gamma_{i} \text { with } \Gamma_{i} \cap \Gamma_{j}=\varnothing, \text { if } i \neq j .
$$

We denote the set of sub-patches as $S(\Gamma)=\left\{\Gamma_{i}\right\}_{i=1}^{N}$.

Without loss of generality, we simply assume a parametric domain $\widehat{D}$ of unit length, i.e., $\widehat{D}=[0,1]^{2}$. For each patch $\Gamma_{i}$, we associate the knot vectors $\mathcal{V}^{i}$ on $\widehat{D}$, which defines a partition $\widehat{\mathcal{T}}_{h, \widehat{D}}^{i}=\left\{\widehat{K}_{m}\right\}_{m=1}^{M_{i}}$, where $\widehat{K}_{m}$ are 2-dimensional closed elements. We refer to $\widehat{\mathcal{T}}_{h, \widehat{D}}^{i}$ as the parametric mesh of patch $\Gamma_{i}$ and denote $\widehat{\mathcal{T}}_{h}=\bigcup_{i=1}^{N} \widehat{\mathcal{T}}_{h, \widehat{D}}^{i}$. Any patch $\Gamma_{i}$ can be represented by a parametrization map as follows

$$
\Phi_{i}: \widehat{D} \rightarrow \Gamma_{i}, \Phi_{i}(\hat{x})=\sum_{j=1}^{N_{i}} C_{j}^{(i)} \widehat{B}_{j}^{(i)}(\hat{x})=x \in \Gamma_{i}
$$

where $C_{j}^{(i)}$ are the control points and $\widehat{B}_{j}^{(i)}$ are B-spline basis functions or NURBS of degree $k$ [18], $N_{i}$ denotes the number of basis functions $\widehat{B}_{j}^{(i)}$ on patch $\Gamma_{i}$.

We obtain the partition $\mathcal{T}_{h, \Gamma_{i}}=\left\{K_{m}\right\}_{m=1}^{M_{i}}$ of the patch $\Gamma_{i}$, whose vertices are the images of the vertices of the corresponding parametric partition $\widehat{\mathcal{T}}_{h, \hat{D}}^{i}$ by the map $\Phi_{i}$. Now we can construct the partition of the domain $\Gamma$, denoted by $\mathcal{T}_{h}$ as follows,

$$
\mathcal{T}_{h}(\Gamma)=\bigcup_{i=1}^{N} \mathcal{T}_{h, \Gamma_{i}}
$$

The set of all the edges of partition $\mathcal{T}_{h}$ is denoted by $\mathcal{E}$ as follows,

$$
\mathcal{E}=\bigcup_{K_{1}, K_{2} \in \mathcal{T}_{h}}\left(\partial K_{1} \cap \partial K_{2}\right) .
$$

We denote the faces of all patches as $\mathcal{F}$ defined by

$$
\mathcal{F}=\bigcup_{i}\left\{e \in \mathcal{E}: e \subset \partial \Gamma_{i}\right\} .
$$

Let $h_{K}$ and $h_{e}$ denote the size of element $K \in \mathcal{T}_{h}$ and the length of the face $e \in \mathcal{E}$ respectively. $h_{\Gamma_{i}}=\max _{K \in \Gamma_{i}}\left\{h_{K}\right\}$ is the mesh size of patch $\Gamma_{i}$. The global mesh size of partition $\mathcal{T}_{h}$ is defined as 


$$
h=\max _{1 \leq i \leq N}\left\{h_{\Gamma_{i}}\right\} .
$$

We assume that the shape of the elements is regular and quasi-uniform, i.e., $h_{e} \sim h$ and $h_{K} \sim h_{\Gamma_{i}}$.

Next, we define the broken space on the physical domain $\Gamma$ associated with $\mathcal{T}_{h}$ by using the introduced push-forward function $\Psi_{i}(x)=\Phi_{i}^{-1}(x)=\hat{x}$, for any $\hat{u} \in H^{s}(\widehat{D})$,

$$
u(x)=\hat{u}\left(\Psi_{i}(x)\right), x \in \Gamma_{i} .
$$

Now we can define the broken Sobolev space $H^{k}\left(\mathcal{T}_{h}\right)$ :

$$
H^{k}\left(\mathcal{T}_{h}\right)=\left\{v \in L^{2}(\Gamma):\left.v\right|_{\Gamma_{i}} \in H^{k}\left(\Gamma_{i}\right), i=1,2, \cdots, N\right\} .
$$

To apply DG method to problem (1), we introduce the jump $\left[u_{h}\right]$ of $u_{h}$ on $F_{i j}$. For $u_{h} \in H^{1}\left(\mathcal{T}_{h}\right)$, using the notation $u_{h}^{i}=\left.u_{h}\right|_{\Gamma_{i}}$, we define

$$
\left[u_{h}\right]=u_{h}^{i} \cdot n_{i}+u_{h}^{j} \cdot n_{j}, \quad \text { on } F_{i j}
$$

where $n_{i}$ denotes the unit normal vector of $\Gamma_{i}$ on $F_{i j}$ pointing exterior to $\Gamma_{j}$.

\subsection{DG-IgA Discretization}

Next, we introduce the finite element space associated with the partition $\mathcal{T}_{h}$. In general, the basis function $B_{j}^{(i)}(x)$ of IgA on the patch $\Gamma_{i}$ are pushed forward from the basis function $\widehat{B}_{j}^{(i)}(\hat{x})$ of the parametric domain $\widehat{D}$ by considering a composition with $\Psi_{i}=\Phi_{i}^{-1}$, i.e., for any basis function $\widehat{B}_{j}^{(i)}$,

$$
B_{j}^{(i)}(x)=\widehat{B}_{j}^{(i)}\left(\Psi_{i}(x)\right) .
$$

The DG finite element space $V_{h}$ is defined by

$$
V_{h}=\left\{u_{h} \in L^{2}\left(\mathcal{T}_{h}\right):\left.u_{h}\right|_{\Gamma_{i}} \subset \operatorname{Span}\left\{B_{j}^{(i)}, j=1, \cdots, N_{i}, i=1, \cdots, N\right\}\right\} .
$$

The DG-IgA approximation is formulated as follows: Find $u_{h} \in V_{h}$ such that

$$
a_{h}\left(u_{h}, v_{h}\right)=F\left(v_{h}\right), \quad \forall v_{h} \in V_{h},
$$

where

$$
\begin{aligned}
a_{h}\left(u_{h}, v_{h}\right) & =\sum_{K \in \mathcal{T}_{h}} \int_{K}\left(-\beta \cdot \nabla_{\Gamma} v_{h}+\gamma v_{h}\right) u_{h} d \sigma+\sum_{e \in \mathcal{E}} \int_{e}\left\{u_{h}\right\}\left[\beta v_{h}\right] d s+\int_{e} \frac{\eta_{e}}{2}\left[u_{h}\right]\left[v_{h}\right] d s, \\
F\left(v_{h}\right) & =\sum_{K \in \mathcal{T}_{h}} \int_{K} f v_{h} d \sigma .
\end{aligned}
$$

where $\eta_{e}$ is the stability parameter, defined as

$$
\eta_{e}= \begin{cases}\frac{1}{2}\left(\left|\beta^{(i)} \cdot n_{i}\right|+\left|\beta^{(j)} \cdot n_{j}\right|\right), & \beta^{(i)}=\beta^{(j)}, \\ |\beta \cdot n|, & \text { otherwise, }\end{cases}
$$

for $e=\partial K_{i} \cap \partial K_{j}$. 
The bilinear map $a_{h}\left(u_{h}, v_{h}\right)$ can be modified. Indeed, by using the continuity of $\beta$ and the function of $V_{h}$ in each patch, i.e., $\left.\left[v_{h}\right]\right|_{\partial K \backslash \partial \Gamma_{i}}=0$ and $\left.\left[\beta v_{h}\right]\right|_{\partial K \backslash \partial \Gamma_{i}}=0$ for any $v_{h} \in V_{h}$, we have

$$
\begin{aligned}
& \sum_{e \in \mathcal{E}} \int_{e}\left[\beta v_{h}\right]\left\{u_{h}\right\} d s+\int_{e} \frac{\eta_{e}}{2}\left[u_{h}\right]\left[v_{h}\right] d s \\
= & \sum_{F_{i j} \in \mathcal{F}} \int_{F_{i j}}\left[\beta v_{h}\right]\left\{u_{h}\right\} d s+\int_{F_{i j}} \frac{\eta_{e}}{2}\left[u_{h}\right]\left[v_{h}\right] d s .
\end{aligned}
$$

Similarly, we can define the outflow faces $\mathcal{F}^{+}$. So, the bilinear map $a_{h}\left(u_{h}, v_{h}\right)$ can be modified as follows

$$
a_{h}\left(u_{h}, v_{h}\right)=\sum_{K \in \mathcal{T}_{h}} \int_{K}\left(-\beta \cdot \nabla_{\Gamma} v_{h}+\gamma v_{h}\right) u_{h} d \sigma+\sum_{F_{i j} \in \mathcal{F}} \int_{F_{i j}}\left[\beta v_{h}\right]\left\{u_{h}\right\} d s+\int_{F_{i j}} \frac{\eta_{e}}{2}\left[u_{h}\right]\left[v_{h}\right] d s .
$$

We can derive the discrete coercivity, stability and consistency of above numerical scheme with a similar technique in [19]. Due to the discontinuity of $\beta$, it needs a few skills to prove the discrete coercivity. So there exists a unique discrete solution $u_{h} \in V_{h}$ satisfying DG-IgA scheme (5). Here we briefly show the a priori error result without detailed proof. We introduce the dual or adjoint weak form: Find $\omega \in H^{s}\left(\mathcal{T}_{h}\right)$ such that

$$
\sum_{K \in \mathcal{T}_{h}}(\omega, \nabla \cdot(\beta v)+\gamma v)_{K}-\sum_{F_{i j} \in \mathcal{F}}\left([\beta v],\left\{\Pi_{h} \omega\right\}\right)_{F_{i j}}+\sum_{F_{i j} \in \mathcal{F}}\left(\frac{\eta_{e}}{2}[v],[\omega]\right)_{F_{i j}}=\left(u_{h}-\Pi_{h} u, v\right),
$$

for all $v \in V(h)$. With the above adjoint weak form, inverse inequality and the approximation of the interpolant operator, we can prove the following result.

Theorem 1. Let $u$ and $u_{h}$ denote the solutions of (1) and (5), respectively. Assume that $\left.u\right|_{K} \in$ $H^{k+1}(K), \omega \in H^{k+1}(K), \forall K \in \mathcal{T}_{h}$. Then we have

$$
\left\|u-u_{h}\right\|_{L^{2}(\Gamma)} \leq C h^{k+1}\|u\|_{H^{k+1}(\Gamma)} .
$$

\section{Numerical Experiments}

In this section, we present some numerical experiments of convection problems on surface. Numerical examples are presented for a sphere and a quarter of a cylinder.

\subsection{Numerical Experiment 1}

We first consider the model problem (1) on the unit sphere

$$
\nabla_{\Gamma} \cdot(\beta u)+\gamma u=f, \quad \text { in } \Gamma
$$

subject to the compatibility condition $\int_{\Gamma} f d \sigma=0$, where $\beta=[1,1,1]^{T}$ and $\gamma=1$. We select the source function $f$ such that the exact solution is $u=\sin (\theta) \sin (\phi)$, where $(\phi, \theta)$ are the spherical coordinates.

We divide the unit sphere $\Gamma$ into 6 patches. For each patch, the knot vector is taken as $[0,0,0,1,1,1] \times[0,0,0,1,1,1]$ to represent the geometry of each patch. We generate the mesh by refining the parameter element of each patch, whose mesh size is denoted by $h$. We show the patches and the uniform meshes of the sphere for $h=1 / 4$ in Figure 1(Left). 

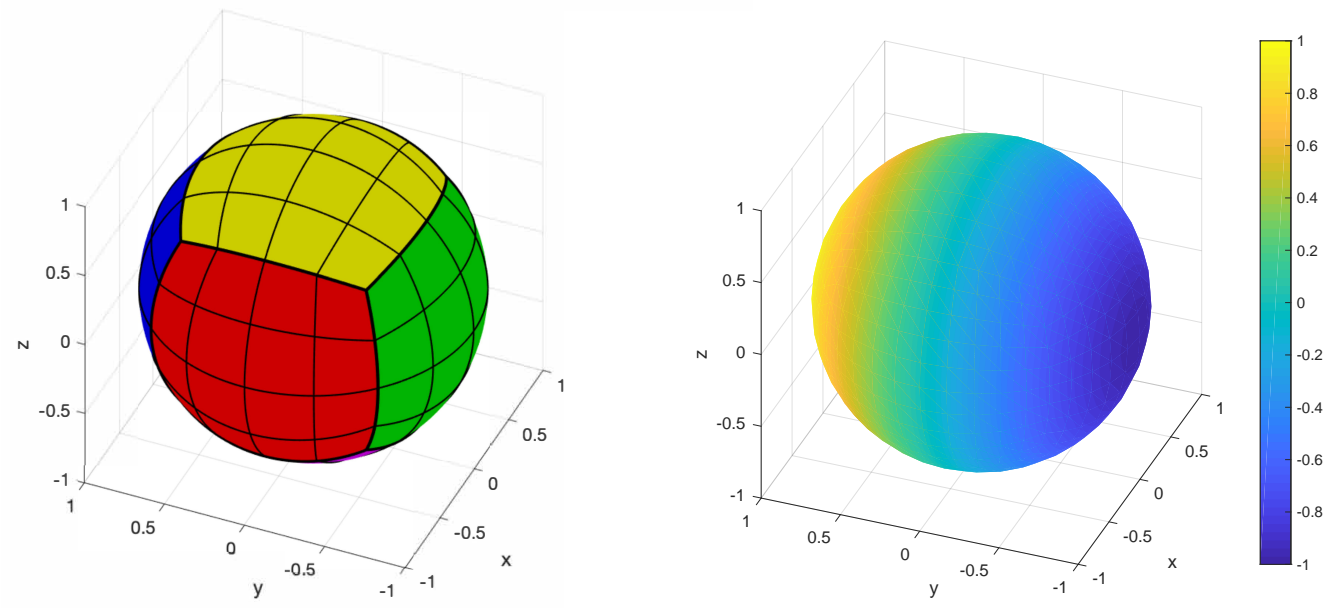

Figure 1. Sphere Case. (Left): The patches and meshes; (Right): Numerical solution.

The numerical $L^{2}$ errors and convergence results are given in Table 1 for $k=1,2$ and 3, respectively. In Figure 2, we present the convergence histories of $L^{2}$ errors. These results show that the IgA-DG method yields $O\left(h^{k+1}\right)$ convergent solution. We present the numerical solution of convection problem (1) on the sphere in Figure 1(Right).

Table 1. Errors and convergence order of sphere case for $k=1,2$ and 3.

\begin{tabular}{cccc}
\hline Degree $\boldsymbol{k}$ & Mesh Size $\boldsymbol{h}$ & Error & Convergence Order \\
\hline 1 & $1 / 2$ & $9.934 \times 10^{-2}$ & - \\
& $1 / 4$ & $1.926 \times 10^{-2}$ & 2.366 \\
& $1 / 8$ & $4.561 \times 10^{-3}$ & 2.078 \\
& $1 / 16$ & $1.128 \times 10^{-3}$ & 2.016 \\
& $1 / 32$ & $2.813 \times 10^{-4}$ & 2.004 \\
\hline 2 & $1 / 2$ & $1.580 \times 10^{-2}$ & - \\
& $1 / 4$ & $1.656 \times 10^{-3}$ & 3.255 \\
& $1 / 8$ & $1.799 \times 10^{-4}$ & 3.202 \\
& $1 / 16$ & $2.202 \times 10^{-5}$ & 3.031 \\
& $1 / 32$ & $2.748 \times 10^{-6}$ & 3.002 \\
\hline 3 & $1 / 2$ & $2.840 \times 10^{-3}$ & - \\
& $1 / 4$ & $3.006 \times 10^{-4}$ & 3.240 \\
& $1 / 8$ & $1.210 \times 10^{-5}$ & 4.635 \\
& $1 / 16$ & $6.826 \times 10^{-7}$ & 4.148 \\
& $1 / 32$ & $4.150 \times 10^{-8}$ & 4.040 \\
\hline
\end{tabular}

\subsection{Numerical Experiment 2}

Here we continue to consider the model problem on the surface of torus

$$
\nabla_{\Gamma} \cdot(\beta u)+\gamma u=f, \quad \text { in } \Gamma,
$$

subject to the compatibility condition $\int_{\Gamma} f d \sigma=0$, where $\beta=[1,1,1]^{T}$ and $\gamma=1$. The torus is the surface

$$
\Gamma=\left\{(x, y) \in(-3,3)^{2}, z \in(-1,1): r^{2}=z^{2}+\left(\sqrt{x^{2}+y^{2}}-R^{2}\right)\right\}
$$


with $r=1$ and $R=2$. We take coordinates $(\phi, \theta)$ as

$$
\phi=\arctan \left(\frac{y}{x}\right), \quad \theta=\arctan \left(\frac{z}{\sqrt{x^{2}+y^{2}}-R}\right),
$$

and select the source function $f$ such that the exact solution is $u=\sin (\theta) \sin (3 \phi)$.

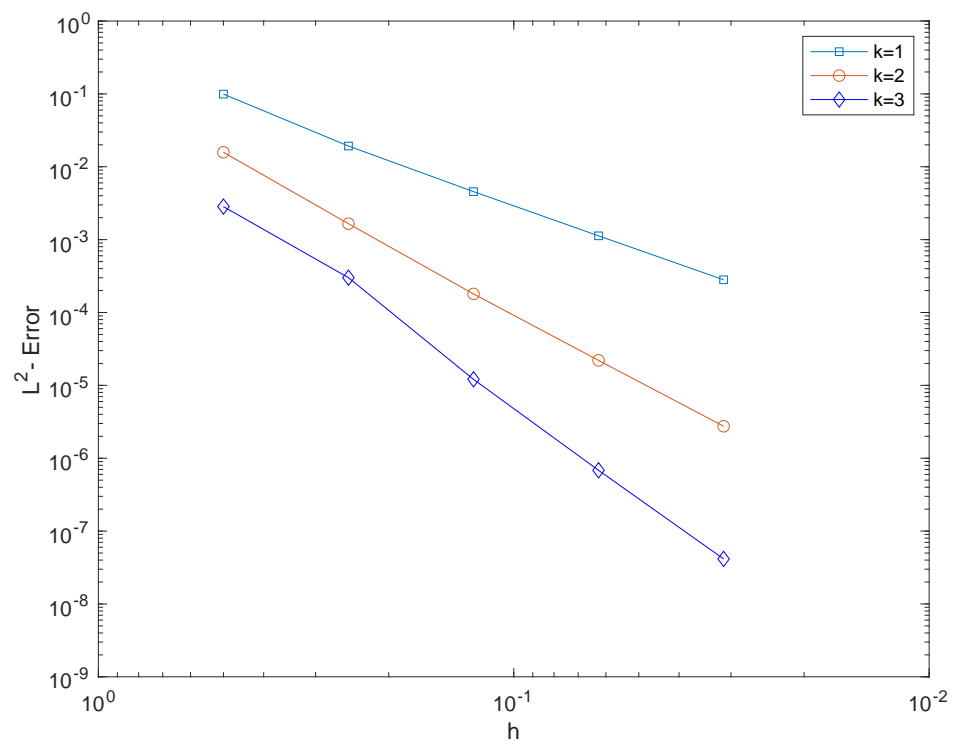

Figure 2. Error convergence result for sphere case.

We divide the torus into 8 patches. For each patch, we take the knot vector as $[0,0,0,1 / 2,1 / 2,1,1,1] \times[0,0,0,1,1,1]$ to give the geometrical representation. We plot the patches and the uniform meshes of the torus for $h=1 / 4$ in Figure 3(Left).

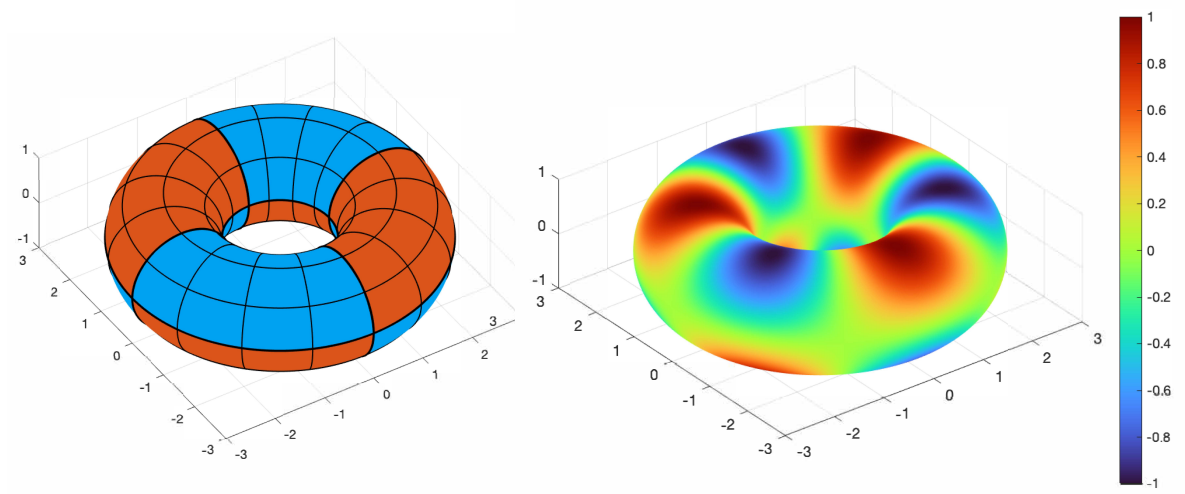

Figure 3. Torus Case. (Left): The patches and meshes; (Right): Numerical solution.

The numerical $L^{2}$ errors and convergence rates of this problem for $k=1,2$ and 3 are shown in Table 2. Table 3 indicate that the rates are also $O\left(h^{k+1}\right)$ for $L^{2}$ norm. Figure 4 shows the convergence history of errors. Finally, we plot the numerical solution of convection problem (1) on torus in Figure 3(Right). 
Table 2. Errors and convergence orders for the torus for $k=1,2$ and 3.

\begin{tabular}{cccc}
\hline Degree $\boldsymbol{k}$ & Mesh Size $\boldsymbol{h}$ & Error & Convergence Order \\
\hline 1 & $1 / 2$ & $2.2825 \times 10^{0}$ & - \\
$1 / 4$ & $3.5096 \times 10^{-1}$ & 2.7013 \\
$1 / 8$ & $7.2088 \times 10^{-2}$ & 2.2835 \\
& $1 / 16$ & $1.7199 \times 10^{-2}$ & 2.0674 \\
& $1 / 32$ & $4.2620 \times 10^{-3}$ & 2.0127 \\
\hline 2 & $1 / 2$ & $9.4780 \times 10^{-1}$ & - \\
& $1 / 4$ & $1.2046 \times 10^{-1}$ & 2.9760 \\
& $1 / 8$ & $9.5761 \times 10^{-3}$ & 3.6530 \\
& $1 / 16$ & $1.1486 \times 10^{-3}$ & 3.0595 \\
& $1 / 32$ & $1.4448 \times 10^{-5}$ & 2.9910 \\
\hline 3 & $1 / 2$ & $0.3400 \times 10^{-1}$ & - \\
& $1 / 4$ & $5.4673 \times 10^{-2}$ & 2.6368 \\
& $1 / 8$ & $9.7914 \times 10^{-4}$ & 5.8032 \\
& $1 / 16$ & $4.6835 \times 10^{-5}$ & 4.3859 \\
& $1 / 32$ & $2.7729 \times 10^{-6}$ & 4.0781 \\
\hline
\end{tabular}

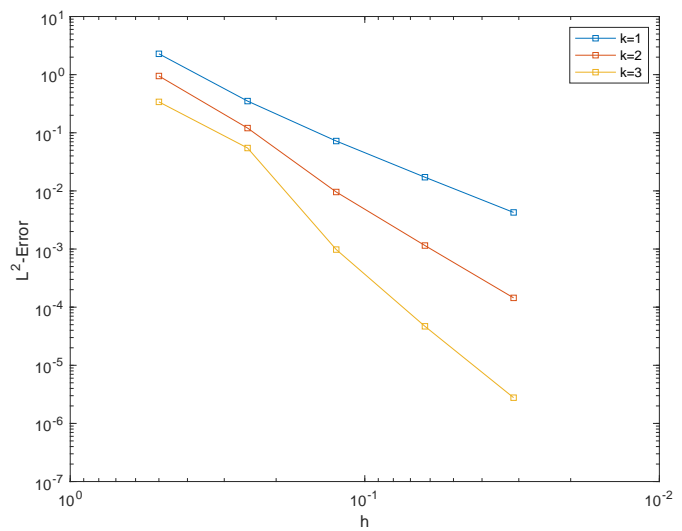

Figure 4. Error convergence result for torus case.

\subsection{Numerical Experiment 3}

Next, we solve on the surface of a square of the cylinder the model problem

$$
\begin{aligned}
\nabla_{\Gamma} \cdot(\beta u)+\gamma u & =f, & & \text { in } \Gamma, \\
u & =g, & & \text { on } \Gamma_{-},
\end{aligned}
$$

where $\gamma=1$. The domain $\Gamma$ is the surface of a quarter of the cylinder, shown in Figure 5. In contrast to the case of sphere, this problem needs the boundary condition $\Gamma_{-}$determined by $\beta$. Then, writing $(r=1, \theta, z)$ to denote the system of cylindrical coordinate, we impose an appropriate boundary condition $g$ for $u$ and source function $f$ so that the exact solution is $u=\sin (z \theta)$.

We consider the model problem on the surface of a square of the cylinder with continuous and discontinuous coefficient $\beta$.

(1) We take continuous coefficient as $\beta=[-1,1,1]^{T}$. We divide the cylinder into 9 patches. For each patch, we take the knot vector as $[0,0,0,1,1,1] \times[0,0,1,1]$ to give the geometrical representation. Similarly, we plot the patches and the uniform meshes of the cylinder for $h=1 / 4$ in Figure 5(Left). 

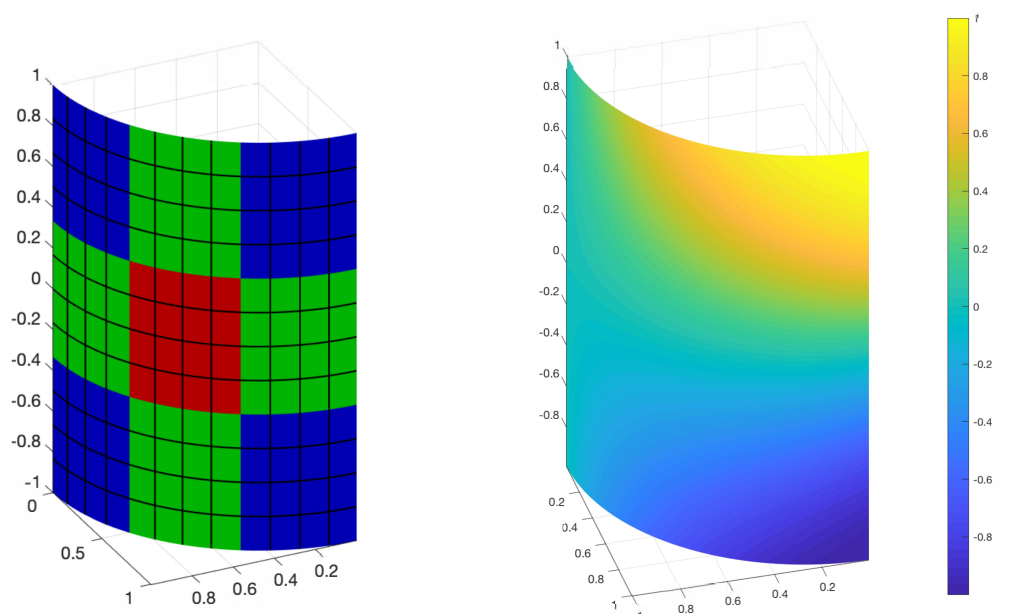

Figure 5. Cylinder Case. (Left): The patches and meshes; (Right): Numerical solution.

Table 3. Errors and convergence order of cylinder case for $k=1,2$ and 3 .

\begin{tabular}{cccc}
\hline Degree $\boldsymbol{k}$ & Mesh Size $\boldsymbol{h}$ & Error & Convergence Order \\
\hline 1 & $1 / 2$ & $7.832 \times 10^{-3}$ & - \\
& $1 / 4$ & $1.912 \times 10^{-3}$ & 2.0344 \\
& $1 / 8$ & $4.748 \times 10^{-4}$ & 2.0097 \\
& $1 / 16$ & $1.185 \times 10^{-4}$ & 2.0023 \\
& $1 / 32$ & $2.962 \times 10^{-5}$ & 2.0004 \\
\hline 2 & $1 / 2$ & $4.5649 \times 10^{-4}$ & - \\
& $1 / 4$ & $5.7310 \times 10^{-5}$ & 2.9937 \\
& $1 / 8$ & $7.2113 \times 10^{-6}$ & 2.9905 \\
& $1 / 16$ & $9.0619 \times 10^{-7}$ & 2.9924 \\
& $1 / 32$ & $1.1364 \times 10^{-7}$ & 2.9954 \\
\hline 3 & $1 / 2$ & $1.7089 \times 10^{-5}$ & - \\
& $1 / 4$ & $1.5120 \times 10^{-6}$ & 3.4986 \\
& $1 / 8$ & $1.0073 \times 10^{-7}$ & 3.9079 \\
& $1 / 16$ & $6.4755 \times 10^{-9}$ & 3.9594 \\
& $1 / 32$ & $4.1048 \times 10^{-10}$ & 3.9796 \\
\hline
\end{tabular}

We present numerical $L^{2}$ errors and convergence rates of this problem for $k=1,2$ and 3 in Table 3. Table 3 indicate that the rates are also $O\left(h^{k+1}\right)$ for $L^{2}$ norm. Figure 6 shows the convergence history of errors. Finally, we plot the numerical solution of convection problem (1) on cylinder in Figure 5(Right). 


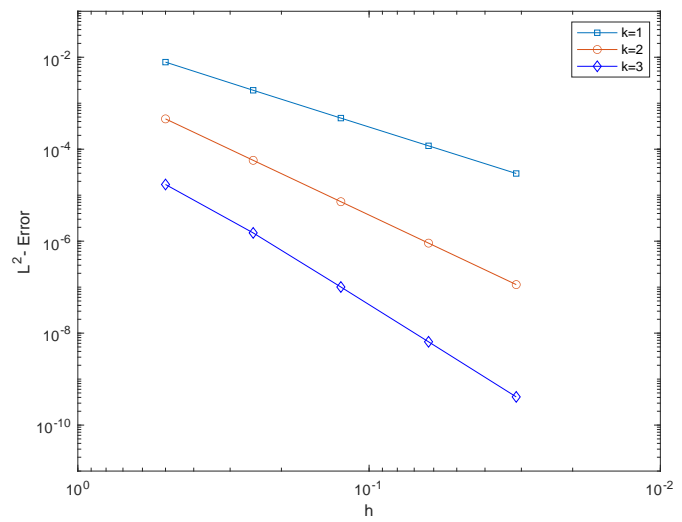

Figure 6. Error convergence result for cylinder case.

(2) We consider the discontinuous advective velocity $\beta$ as

$$
\begin{aligned}
& \beta^{(1)}=\beta^{(4)}=[-1 ; 1 ; 1], \\
& \beta^{(2)}=\beta^{(3)}=[-2 ; 4 ; 3],
\end{aligned}
$$

where the index $(i)$ is the patch number. Observe that the source function is discontinuous across the patches according to the choice of $\beta$.

According to the discontinuity of $\beta$, we divide the cylinder into 4 patches. For each patch, we take the knot vector as $[0,0,0,1,1,1] \times[0,0,1,1]$ to give the geometrical representation. Similarly, we plot the patches and the uniform meshes of the cylinder for $h=1 / 4$ in Figure 7(Left), where patch 1 and patch 4 are painted blue, and patch 2, 3 are painted yellow.
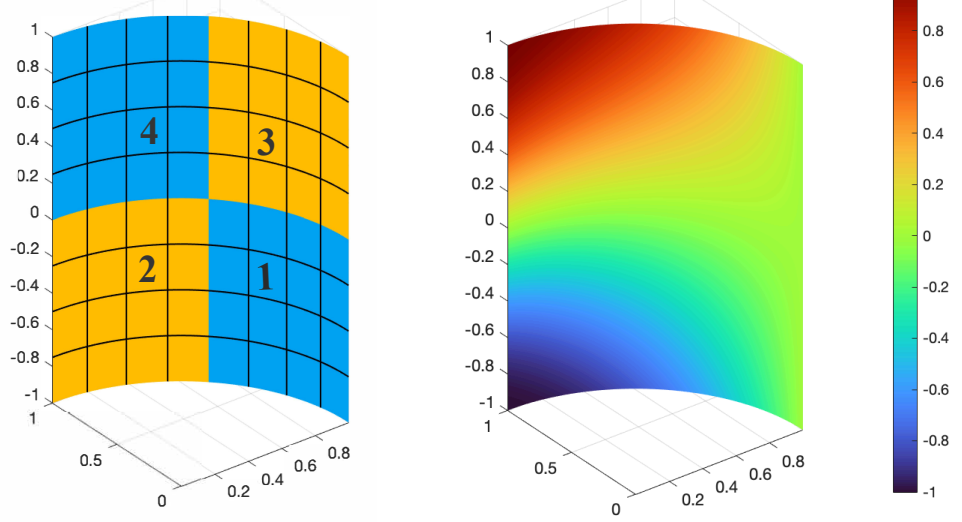

Figure 7. Cylinder Case. (Left): The patches and meshes; (Right): Numerical solution.

The numerical $L^{2}$ errors and convergence rates of this problem for $k=1,2$ and 3 are shown in Table 4. Table 4 indicate that the rates are also $O\left(h^{k+1}\right)$ for $L^{2}$ norm. Figure 8 shows the convergence history of errors. Finally, we plot the numerical solution of convection problem (1) on cylinder in Figure 7(Right). 
Table 4. Errors and convergence orders for the cylinder for $k=1,2$ and 3.

\begin{tabular}{cccc}
\hline Degree $\boldsymbol{k}$ & Mesh Size $\boldsymbol{h}$ & Error & Convergence Order \\
\hline 1 & $1 / 2$ & $1.774 \times 10^{-2}$ & - \\
& $1 / 4$ & $4.277 \times 10^{-3}$ & 2.0531 \\
$1 / 8$ & $1.063 \times 10^{-3}$ & 2.0085 \\
& $1 / 16$ & $2.656 \times 10^{-4}$ & 2.0006 \\
& $1 / 32$ & $6.640 \times 10^{-5}$ & 2.0002 \\
\hline 2 & $1 / 2$ & $1.7036 \times 10^{-3}$ & - \\
& $1 / 4$ & $1.9959 \times 10^{-4}$ & 3.0935 \\
& $1 / 8$ & $2.4770 \times 10^{-5}$ & 3.0104 \\
& $1 / 16$ & $3.1024 \times 10^{-6}$ & 2.9971 \\
& $1 / 32$ & $3.8885 \times 10^{-7}$ & 2.9961 \\
\hline 3 & $1 / 2$ & $1.0065 \times 10^{-4}$ & - \\
& $1 / 4$ & $8.1947 \times 10^{-6}$ & 3.6186 \\
& $1 / 8$ & $5.3064 \times 10^{-7}$ & 3.9489 \\
& $1 / 16$ & $3.3827 \times 10^{-8}$ & 3.9715 \\
& $1 / 32$ & $2.1381 \times 10^{-9}$ & 3.9838 \\
\hline
\end{tabular}

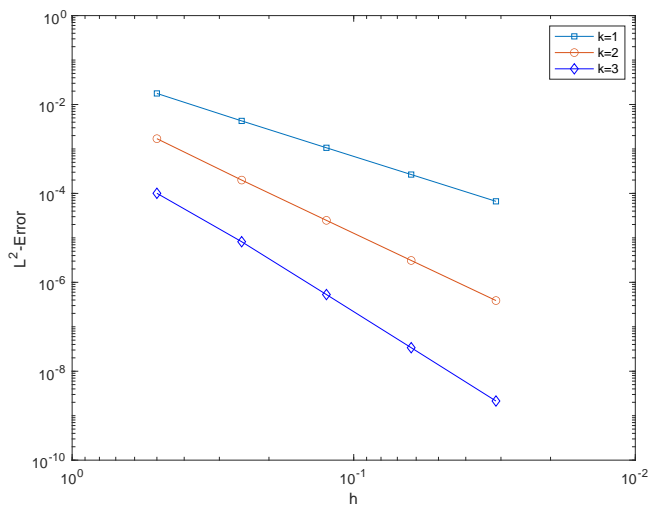

Figure 8. Error convergence result for cylinder case.

\section{Conclusions}

In this paper, we present the new penalty discontinuous Galerkin (DG) isogeometric analysis(IgA) methods to solve convection problems with continuous or discontinuous coefficient on implicitly defined surfaces. For further purpose, it is worthy studying the stability and error analysis of this method and more practical problems on surfaces.

Author Contributions: Conceptualization and methodology, C.X.; formal analysis, software and validation, L.W.; visualization, X.Y.; writing-review and editing, H.W. All authors have read and agreed to the published version of the manuscript.

Funding: This research was funded by the National Basic Research and Development Program of China grant number 2017YFC1502201, NSFC grant number 11672032, NSFC grant number 10871218 and NSFC grant number 61873036.

Institutional Review Board Statement: Not applicable.

Informed Consent Statement: Not applicable.

Data Availability Statement: Not applicable.

Conflicts of Interest: The authors declare no conflict of interest. 


\section{References}

1. Milliken, W.J.; Stone, H.A.; Leal, L.G. The effect of surfactant on transient motion of newtonian drops. Phys. Fluids A 1993, 5, 69-79. [CrossRef]

2. Elliott, C.M.; Stinner, B. Modeling and computation of two phase geometric biomembranes using surface finite elements. J. Comput. Phys. 2010, 229, 6585-6612. [CrossRef]

3. Dziuk, G. Finite elements for the Beltrami operator on arbitrary surfaces. Partial differential equations and calculus of variations. Lect. Notes Math. 1988, 1357, 142-155.

4. Bonito, A.; Pasciak, J.E. Convergence analysis of variational and non-variational multi-grid algorithms for the Laplace-Beltrami operator. Math. Comp. 2012, 81, 1263-1288. [CrossRef]

5. Houston, P.; Schwab, C.; Süli, E. Discontinuous hp-finite element methods for advection-diffusion-reaction problems. SIAM J Numer. Anal. 2002, 39, 2133-2163. [CrossRef]

6. Xiong, C.; Li, Y. Error analysis for optimal control problem governed by convection diffusion equations: DG method. J. Comput. Appl. Math. 2011, 235, 3163-3177. [CrossRef]

7. Xiong, C.; Luo, F.; Ma, X.; Li, Y. A priori error analysis for optimal distributed control problem governed by the first order linear hyperbolic equation: hp-streamline diffusion discontinuous galerkin method. J. Numer. Math. 2016, 24, 125-134. [CrossRef]

8. Xiong, C.; Li, Y. A posteriori error estimators for optimal distributed control governed by the first-order linear hyperbolic equation: DG method. NMPDEs 2011, 27, 491-506. [CrossRef]

9. Xiong, C.; Becker, R.; Luo, F.; Ma, X. A priori and a posteriori error analysis for the mixed discontinuous Galerkin finite element approximations of the biharmonic problems. NMPDEs 2017, 33, 318-353. [CrossRef]

10. Wang, L.; Xiong, C.; Wu, H.; Luo, F. A priori and a posteriori error analysis for discontinuous Galerkin finite element approximations of biharmonic eigenvalue problems. Adv. Comput. Math. 2013, 45, 2623-2646. [CrossRef]

11. Dedner, A.; Madhavan, P. Discontinuous Galerkin Methods for Hyperbolic and Advection-Dominated Problems on Surfaces. 2015. Available online: https:/ /arxiv.org/pdf/1505.06752.pdf (accessed on 20 December 2020).

12. Hughes, T.J.R.; Cottrell, J.A.; Bazilevs, T. Isogeometric analysis: CAD, finite elements, NURBS, exact geometry and mesh refinement. Comput. Methods Appl. Mech. Engrg. 2005, 194, 4135-4195. [CrossRef]

13. Langer, U.; Toulopoulos, I. Analysis of multipatch discontinuous Galerkin IgA approximations to elliptic boundary value problems. Comput. Vis. Sci. 2015, 17, 217-233. [CrossRef]

14. Zhang, F.; Xu, Y.; Chen, F. Discontinuous Galerkin methods for isogeometric analysis for elliptic equations on surfaces. Commun. Math. Stat. 2014, 2, 431-461. [CrossRef]

15. Hofer, C.; Langer, U.; Toulopoulos, I. Discontinuous Galerkin Isogeometric Analysis of Elliptic Diffusion Problems on Segmentations with Gaps. Comput. Math. Appl. 2015, 72, 1811-1827. [CrossRef]

16. Bazilevs, Y.; Hughes, T.J.R. NURBS-based isogeometric analysis for the computation of flows about rotating components. Comput. Mech. 2008, 43, 143-150. [CrossRef]

17. Elfverson, D.; Larson, M.G.; Larsso, K. A new least squares stabilized Nitsche method for cut isogeometric analysis. Comput. Methods Appl. Mech. Eng. 2019, 349, 1-16. [CrossRef]

18. Schumaker, L.L. Spline Functions: Basic Theory, 3rd ed.; Cambridge University Press: Cambridge, UK, 2007.

19. Brezzi, F.; Marini, L.D.; Süli, E. Discontinuous Galerkin methods for first-order hyperbolic problems. Math. Model. Methods Appl. Sci. 2004, 14, 1893-1903. [CrossRef] 